\title{
SerPens - A Tool for Semantically Enriched Location Information on Personal Devices
}

\author{
Sourav Bhattacharya, Joonas Kukkonen, Petteri Nurmi and Patrik Floréen \\ Helsinki Institute for Information Technology HIIT \\ Department of Computer Science, University of Helsinki \\ P. O. Box 68, Fl-00014 University of Helsinki, Finland \\ firstname.lastname@cs.helsinki.fi
}

\begin{abstract}
Current mobile phones provide GSM cell information and many devices also support GPS or WiFi-based location information. A problem with raw location data is that it does not provide semantic information, which makes it hard to integrate location-awareness into applications. Moreover, to understand what kind of location information is important to users, researchers currently need to perform time consuming user studies. In this paper we introduce SerPens, a tool that enables gathering semantically enriched location information on personal devices. The main novelty of SerPens is that it enables users to share and gather semantic information in a collaborative fashion. The label information is tied to a taxonomy and is accessible to applications. SerPens has been developed on top of BeTelGeuse, a Bluetooth-based data gathering tool for J2ME compatible devices.
\end{abstract}

\section{Keywords}

location-awareness, location semantics, data gathering

\section{INTRODUCTION}

Location information remains one of the most important sources of contextual information. Location often serves as a good indicator of the user's situation and some form of location information is easily accessible on current mobile devices. For example, most mobile phones provide GSM cell information and an increasing amount of devices support GPS or WiFi-based location information.

A problem with raw location information is that it provides little or no semantic meaning. For example, GPS and GSM data are numeric in nature, whereas humans refer to locations using semantic descriptions, e.g., home, Starbucks or University [7]. Furthermore, location-aware applications require a mapping from spatial coordinates to objects of interest; e.g., a location-aware restaurant guide requires information about the coordinates of restaurants.

We present SerPens, a tool for semantically enriched location information on personal devices. SerPens allows users

Permission to make digital or hard copies of all or part of this work for personal or classroom use is granted without fee provided that copies are not made or distributed for profit or commercial advantage and that copies bear this notice and the full citation on the first page. To copy otherwise, to republish, to post on servers or to redistribute to lists, requires prior specific permission and/or a fee.

Copyright 200X ACM X-XXXXX-XX-X/XX/XX ...\$5.00. to associate semantics using different granularities and different location sources. The semantics are tied to a taxonomy which facilitates analysis of location information. SerPens supports gathering generic (e.g., street or district) and personally meaningful semantics. The generic information can be shared among different users in collaborative fashion whereas personally meaningful information is private. We have developed SerPens on top of BeTelGeuse, a Bluetooth data gathering tool for J2ME compatible devices [5].

\section{RELATED WORK}

Intel's Placelab initiative is a good example of earlier approaches to gathering location information in a collaborative fashion [6]. Also approaches that map locations of GSM cell towers have been introduced (e.g., GSMLoc ${ }^{1}$ ). Hariharan et al. [2] use a commercial geocoder to map GPS information into street addresses. Li et al. [3] associate semantics to individual GSM cells. To find out what kind of locations are important, various user studies have been carried out. For example, Zhou et al. [8] compare automatically extracted information to user's daily visited place information maintained in a diary. This method is very time consuming. With SerPens users need to label the locations once and the information they provide can be compared with the information that other users have provided about nearby locations. Furthermore, SerPens provides a reliable way to associate semantics to different granularities of location information.

\section{SYSTEM OVERVIEW}

SerPens has been implemented using the client-server architecture. The client acts as a proxy for the communications between the mobile device and the server whereas the server manages the mappings between location information and semantics. The server can also refine location information using third party services, e.g., commercial geocoders.

\subsection{BeTelGeuse}

Our location gathering system is built on top of the BeTelGeuse $^{2}$ Bluetooth data gathering tool [5]. BeTelGeuse turns a mobile device, such as a mobile phone, into a relay node that collects data from a body area network over Bluetooth. Depending on the device, BeTelGeuse can provide GSMbased and GPS-based location information to SerPens. For example, on Nokia S60 3rd edition phones we use Python to read GSM cell information from the phone.

\footnotetext{
${ }^{1}$ www.gsmloc.org [Retrieved: 2007-09-30]

${ }^{2}$ http://www.cs.helsinki.fi/group/acs/betelgeuse/
} 


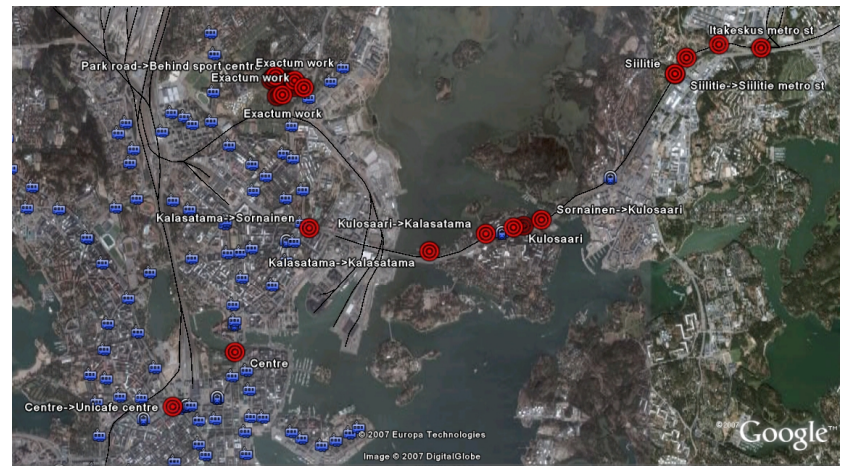

Figure 1: Visualization of semantic information associated with location data.

\subsection{Mobile Client}

The main purpose of the client is to collect data from the mobile device and to send the data to the server. As different users often interpret the same location differently [7], the client program also enables users to add semantics to different granularities. The different granularities are specified by a location taxonomy that currently supports three kinds of locations: geographic locations (street, address etc.), places (home, university, park) and GSM cell specific information. Of particular interest to us is the place category. The labels provided by the users can be matched with results from location clustering (see Sec. 4) and hence we do not have to request users to assign names for detected clusters. The taxonomy for place information is based on results of a user study that examined how people describe places [9].

\subsection{Server}

The server side is responsible for maintaining associations between the semantics and the location information. We also maintain information about the user who provided the semantics, this way we can support both private and public semantic labels. When a user provides new semantic information, the client sends the semantics and all available location information to the server. The server then stores the information in a spatial database. Later on, when the client queries for semantics, we can use neighborhood queries on the spatial database to return relevant semantics.

The server is also responsible for analyzing location data and for communicating with third party services. For example, the server can cluster data or connect to an external geocoding service. On top of the server we have implemented a visualization interface using Google Earth; see Fig. 1.

\section{USAGE SCENARIOS}

To illustrate the usefulness of SerPens, this section gives some examples of how SerPens can be used to facilitate research and to support applications.

Location clustering: Location clustering attempts to discover places that are important to a user from raw location data [4]. The labels that the users provide serve as a ground truth for validating the performance of different algorithms.

Automated diaries: Recently, several approaches for generating automatically diaries or blogs about daily ac- tivities have been suggested [1]. SerPens can be used as an information source that automatically provides semantics for the locations that a user has visited.

Geographic web: The geographic web refers to complimenting location measurements with information from the Internet [2]. Currently, the only way to support the geographic web is to use commercial geocoders that transform GPS coordinates into street addresses. With SerPens, the geographic web can be supported when GPS information is not available and queries can be performed using different granularities of information.

\section{REFERENCES}

[1] S.-B. Cho, K.-J. Kim, K. S. Hwang, and I.-J. Song. AniDiary: Cartoon-style diary exploits Bayesian networks. IEEE Pervasive Computing, 6(3):66-75, 2007.

[2] R. Hariharan, J. Krumm, and E. Horvitz. Web-enhanced GPS. In T. Strang and C. Linnhoff-Popien, editors, Proceedings of the 1st International Workshop on Location and Context-Awareness (LoCA), volume 3479 of Lecture Notes in Computer Science, pages 95-104. Springer, 2005.

[3] W. Li and M. Jonsson. Providing user self-contained location information using mobile phones. In Adjunct Proceedings of the 4th International Conference on Pervasive Computing (PERVASIVE), pages 33-36, 2006.

[4] P. Nurmi and J. Koolwaaij. Identifying meaningful locations. In Proceedings of the 3rd International Conference on Mobile and Ubiquitous Computing (MobiQuitous). IEEE Computer Society, 2006.

[5] P. Nurmi, J. Kukkonen, E. Lagerspetz, J. Suomela, and P. Floréen. BeTelGeuse - a tool for Bluetooth data gathering. In Proceedings of the 2nd International Conference on Body Area Networks (BodyNets). ACM, 2007.

[6] T. Sohn, W. G. Griswold, J. Scott, A. LaMarca, Y. Chawathe, I. Smith, and M. Y. Chen. Experiences with Place Lab: An open source toolkit for locaton-aware computing. In Proceedings of the 28th International Conference on Software engineering, pages 462-471. ACM Press, 2006.

[7] A. H. Weilenmann and P. Leuchovius. "I'm waiting where we met last time": Exploring everyday positioning practices to inform design. In R. Raisamo, editor, Proceedings of the 3rd Nordic Conference on Human-Computer Interaction (NordiCHI), pages 33-42. ACM, 2004.

[8] C. Zhou, D. Frankowski, P. Ludford, S. Shekhar, and L. Terveen. Discovering personally meaningful places: An interactive clustering approach. ACM Transactions on Information Systems, 25(3):12, 2007.

[9] C. Zhou, P. Ludford, D. Frankowski, and L. Terveen. Talking about places: An experiment in how people describe places. In Adjunct Proceedings of the 3rd International Conference on Pervasive Computing (PERVASIVE), pages 37-41, 2005. 